\title{
Olfactomedin 4 (GW112, hGC-1) is an independent prognostic marker for survival in patients with colorectal cancer
}

\author{
NAOTSUGU SEKO ${ }^{1}$, NAOHIDE OUE ${ }^{1}$, TSUYOSHI NOGUCHI ${ }^{3}$, KAZUHIRO SENTANI $^{1}$, \\ NAOYA SAKAMOTO $^{1}$, TAKAO HINOI ${ }^{2}$, MASAZUMI OKAJIMA ${ }^{2}$ and WATARU YASUI ${ }^{1}$ \\ Departments of ${ }^{1}$ Molecular Pathology, and ${ }^{2}$ Endoscopic Surgery and Surgical Science, \\ Graduate School of Biomedical Sciences, Hiroshima University, Hiroshima; \\ ${ }^{3}$ Department of Gastrointestinal Surgery, Oita University Faculty of Medicine, Oita, Japan
}

Received August 28, 2009; Accepted October 26, 2009

DOI: 10.3892/etm_00000013

\begin{abstract}
Colorectal cancer (CRC) is one of the leading causes of cancer-related deaths worldwide. We previously performed Serial Analysis of Gene Expression (SAGE) on four primary gastric cancer samples and identified several gastric cancer-specific genes. Of these genes, olfactomedin 4 (OLFM4, also known as GW112 or hGC-1) is a candidate gene for cancer-specific expression. In the present study, we examined the expression and distribution of olfactomedin 4 in $\mathrm{CRC}$ by immunohistochemistry. Of the $176 \mathrm{CRC}$ cases, 59 (34\%) were positive for cytoplasmic staining of olfactomedin 4. Olfactomedin 4-positive CRC cases showed earlier T classification $(\mathrm{P}=0.0180), \mathrm{N}$ classification $(\mathrm{P}=0.0149)$ and stage $(\mathrm{P}=0.0144)$ than olfactomedin 4-negative $\mathrm{CRC}$ cases. In the $176 \mathrm{CRC}$ patients, those with olfactomedin 4-positive CRC had a better survival rate than patients with olfactomedin 4-negative CRC $(\mathrm{P}=0.0092)$. Multivariate analysis indicated that $\mathrm{T}$ classification, $\mathrm{M}$ classification and negative olfactomedin 4 expression were independent predictors of survival in patients with CRC. In addition to cytoplasmic staining of olfactomedin 4 , stromal staining at the invasive front was observed. In total, 29 (16\%) of the 176 CRC cases were positive for stromal olfactomedin 4; however, stromal olfactomedin 4 staining was not correlated with any clinicopathologic characteristic or with patient survival. These results indicate that olfactomedin 4 is a valuable marker for long-term survival in patients with CRC.
\end{abstract}

\section{Introduction}

Colorectal cancer (CRC) is one of the leading causes of cancer-related death worldwide. An assessment of prognosis based on features of the resected tumor would permit treating

Correspondence to: Dr Wataru Yasui, Department of Molecular Pathology, Hiroshima University Graduate School of Biomedical Sciences, 1-2-3 Kasumi, Minami-ku, Hiroshima 734-8551, Japan E-mail:wyasui@hiroshima-u.ac.jp

Key words: olfactomedin 4, OLFM4, colorectal cancer, prognosis physicians to qualify the benefit of adjuvant chemotherapy to individual patients. Currently, anatomic and pathologic staging is still the most accurate predictor of patient outcome. It would be valuable to supplement standard clinical and pathologic staging using molecular markers to more precisely define the subset of patients at highest or lowest risk of relapse following CRC surgery. This would facilitate better selection of patients who would benefit most from adjuvant therapy. One of the most promising molecular markers is the presence of tumor microsatellite instability (1). We previously reported that expression of Reg IV and h-prune are prognostic makers for CRC (2,3); however, these markers cannot completely identify which patients are at low or high risk for disease recurrence. Therefore, identification of better prognostic markers for patients with CRC is important.

We previously performed Serial Analysis of Gene Expression (SAGE) on four primary gastric cancer samples (4) and identified several gastric cancer-specific genes (5). Of these genes, olfactomedin 4 (OLFM4, also known as GW112 or hGC-1) is a candidate gene for cancer-specific expression, at least in patients with gastric cancer. OLFM4 was originally cloned from human hematopoietic myeloid cells (6). Although OLFM4 is predominantly expressed in bone marrow, the small intestine, colon and prostate (6), levels of expression are much lower in normal tissues than in gastric cancer tissues (5). Enhanced olfactomedin 4 expression has been reported in gastric cancer by Northern blot analysis (7) and by immunostaining (8). Our previous immunohistochemical analysis revealed that olfactomedin 4 is expressed in 56\% of gastric cancer tissues (9). In addition, olfactomedin 4 is a secreted protein, and we showed that serum olfactomedin 4 represents a novel biomarker for gastric cancer (9). In CRC patients, preoperative serum levels of olfactomedin 4 were increased in a small number of samples, and the sensitivities of serum olfactomedin 4 at stage I-III were lower than those of CEA (9).

In addition to gastric cancer, OLFM4 mRNA and olfactomedin 4 protein overexpression have been reported in CRC $(10,11)$. Olfactomedin 4 inhibits apoptosis and may have significant roles in the development of cancer (7). It has been proposed that olfactomedin 4 can serve as a useful marker for stem cells in the human small intestine and colon (12). In 
contrast to these observations, immunohistochemical analysis has demonstrated that olfactomedin 4 down-regulation is found in late stage CRC cases and in CRC patients with shorter survival (13). The morphology and actin distribution of the HT-29 CRC cell line was altered by forced expression of olfactomedin 4. Forced expression of olfactomedin 4 did not change cell proliferation, but decreased cell adhesion and migration (13). Our previous immunohistochemical analysis in gastric cancer revealed that patients with olfactomedin 4-positive gastric cancer had a better survival rate than patients with olfactomedin 4-negative gastric cancer. These results suggest that olfactomedin 4 can inhibit tumor progression. Thus, the clinical significance of olfactomedin 4 expression in human cancers is controversial and still unclear.

Although immunohistochemical analysis of olfactomedin 4 has been performed in CRC (13), this study was performed using tissue microarray. Therefore, detailed expression and distribution of olfactomedin 4 in CRC has not yet been investigated. In the present study, we examined the expression and distribution of olfactomedin 4 in CRC by immunohistochemistry and the relationship between olfactomedin 4 staining and clinicopathologic characteristics.

\section{Materials and methods}

Tissue samples. In a retrospective study design, 176 primary tumors were collected from patients diagnosed with CRC who underwent surgery at Hiroshima University Hospital (Hiroshima, Japan). All patients underwent curative resection. Only patients without preoperative radiotherapy or chemotherapy were enrolled in the study. The patients were comprised of 105 men and 71 women. The mean age was 63 years (range, 29-89 years). Postoperative follow-up was scheduled every 1,2 or 3 months during the first 2 years after surgery and every 6 months thereafter, unless more frequent follow-up was deemed necessary. Chest X-rays, chest computed tomography scans and serum chemistries were performed at every follow-up visit. Recurrence was evaluated from records at Hiroshima University Hospital. For immunohistochemical analysis, we used archival formalin-fixed, paraffin-embedded tissues. Histologic classification was based on the World Health Organization system. Tumor staging was performed according to the TNM stage grouping system (14). Since written informed consent was not obtained, for strict privacy protection, identifying information for all samples was removed before analysis; this procedure is in accordance with the Ethical Guidelines for Human Genome/Gene Research enacted by the Japanese Government.

Immunohistochemistry. From each patient, one or two representative tumor blocks, including the tumor center, invading front and tumor-associated non-neoplastic mucosa, were examined by immunohistochemistry. In cases of large, late-stage tumors, two different sections were examined to include representative areas of the tumor center as well as of the lateral and deep tumor invasive front. Olfactomedin 4 was detected immunohistochemically with a monoclonal antibody raised in our laboratory (9). The specificity of the anti-olfactomedin 4 antibody has been characterized in detail (9). A Dako Envision+ Mouse Peroxidase Detection System
(Dako Cytomation, Carpinteria, CA, USA) was used for immunohistochemical analysis as described previously (9). In brief, antigen retrieval was carried out by microwave heating in citrate buffer ( $\mathrm{pH} \mathrm{6.0)}$ ) for $30 \mathrm{~min}$. After peroxidase activity was blocked with $3 \% \mathrm{H}_{2} \mathrm{O}_{2}$-methanol for $10 \mathrm{~min}$, sections were incubated with normal goat serum (Dako Cytomation) for 20 min to block nonspecific antibody binding sites. Sections were incubated with primary antibody against olfactomedin 4 (1:50) for $1 \mathrm{~h}$ at room temperature, followed by incubations with Envision+ anti-mouse peroxidase for $1 \mathrm{~h}$. Staining was completed with a 10-min incubation with the substratechromogen solution. Sections were counterstained with $0.1 \%$ hematoxylin. Negative controls were created by omission of the primary antibody.

Statistical methods. Correlations between clinicopathologic parameters and olfactomedin 4 expression were analyzed by the Chi-square test. Kaplan-Meier survival curves were constructed for olfactomedin 4-positive and olfactomedin 4-negative patients. Survival rates were compared between olfactomedin 4-positive and olfactomedin 4-negative groups. Differences between survival curves were tested for statistical significance by the log-rank test (15). The Cox proportional hazards multivariate model was used to examine the association of clinical and pathologic factors and the expression of olfactomedin 4 with survival. A P-value of $<0.05$ was considered statistically significant.

\section{Results}

Expression and distribution of olfactomedin 4 in CRC and peritumoral mucosa. We performed immunohistochemical analysis of olfactomedin 4 in 176 human CRC samples. In $\mathrm{CRC}$ tissue, olfactomedin 4 staining was frequently observed in well-differentiated (Fig. 1A) and moderately differentiated adenocarcinoma (Fig. 1B). In general, staining for olfactomedin 4 was detected in the cytoplasm of tumor cells. The percentage of olfactomedin 4-stained tumor cells ranged from 0 to $80 \%$. It has been reported that a loss/ reduction in olfactomedin 4 expression at the front of the invasion is observed in CRC (13); however, the tendency for loss of olfactomedin 4 expression at the invasive front was not observed. In our previous immunohistochemical analysis of gastric cancer (9), in addition to cytoplasmic staining, extracellular staining of olfactomedin 4 was observed. In CRC tissues, extracellular staining of olfactomedin 4 was also observed. Extracellular staining of olfactomedin 4 was focal, and in general, extracellular staining of olfactomedin 4 was observed at the invasive front (Fig. 1C). The immunoreactivity for olfactomedin 4 was irregular and fibrous around tumor cells scattered in the stroma (Fig. 1D).

We then focused on the peritumoral mucosa of CRC. Notably, strong and extensive olfactomedin 4 staining was detected, and all peritumoral mucosa samples in the $176 \mathrm{CRC}$ cases were positive for olfactomedin 4 regardless of the olfactomedin 4 staining in tumor cells. Olfactomedin 4 staining decreased gradually, moving away from the CRC tissue. In the mucosa closest to the tumor tissue, almost all epithelial cells showed olfactomedin 4 staining (Fig. 1E). In contrast, in the mucosa distant from the tumor tissue, few epithelial cells 

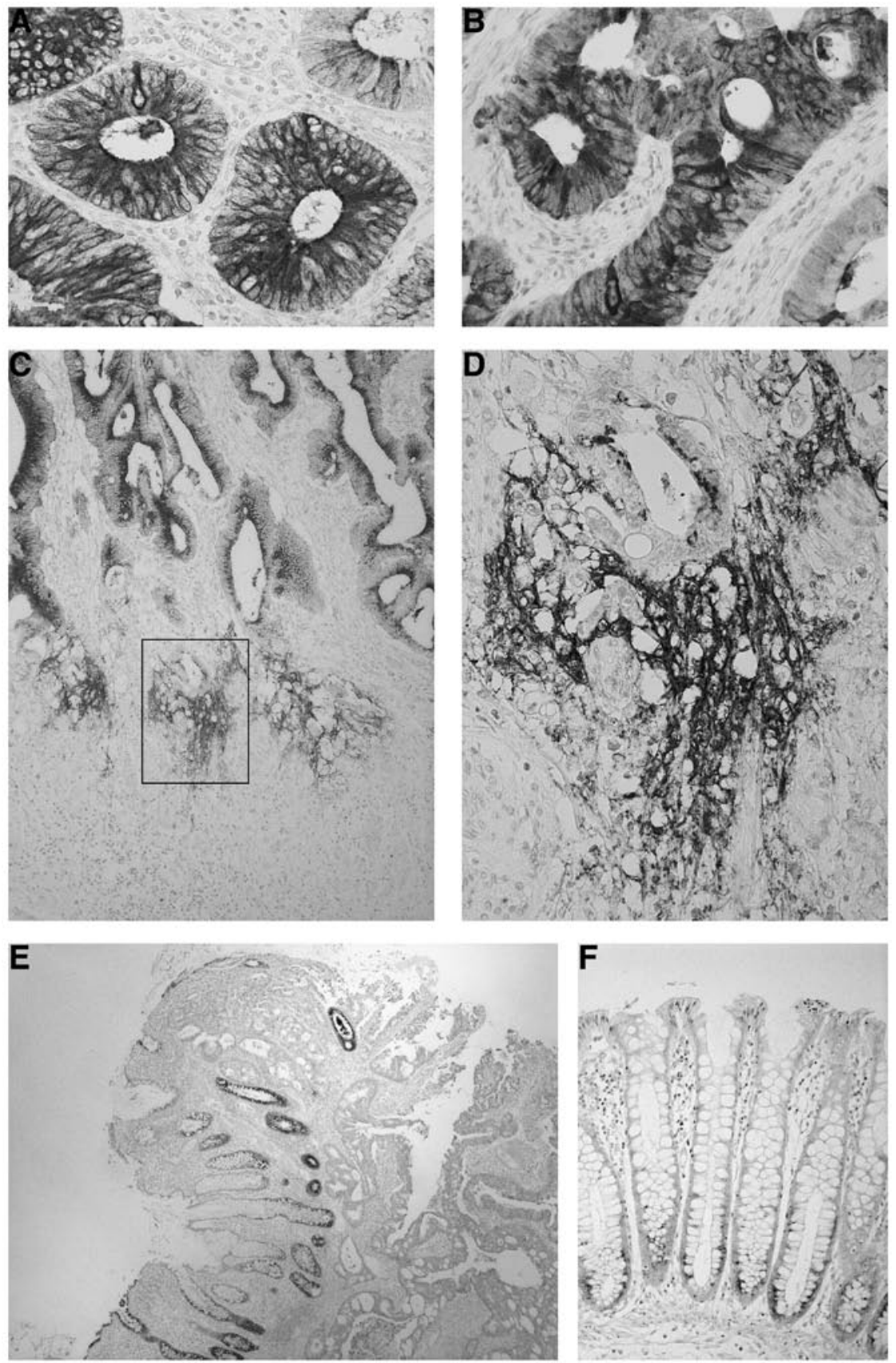

Figure 1. Immunohistochemical analysis of olfactomedin 4 in CRC and peritumoral mucosa. (A) Immunostaining of olfactomedin 4 in well-differentiated CRC. Staining of olfactomedin 4 was detected in the cytoplasm of tumor cells. Original magnification x400. (B) Immunostaining of olfactomedin 4 in moderately differentiated CRC. Staining of olfactomedin 4 was detected in the cytoplasm of tumor cells. Original magnification x400. (C) Immunostaining of olfactomedin 4 in CRC. Extracellular staining of olfactomedin 4 was observed at the invasive front. Original magnification x100. (D) High-magnification image of the field indicated by the box in C. The immunoreactivity for olfactomedin 4 was irregular and fibrous around tumor cells scattered in the stroma. Original magnification $\mathrm{x} 400$. (E) Immunostaining of olfactomedin 4 in the peritumoral mucosa of CRC. Almost all epithelial cells showed olfactomedin 4 staining. Original magnification $x$ 40. (F) Immunostaining of olfactomedin 4 in the mucosa distant from the tumor tissue. Few epithelial cells showed olfactomedin 4 staining. Original magnification $\times 200$.

showed olfactomedin 4 staining (Fig. 1F). Olfactomedin 4 was expressed in the basal crypt epithelium in the colon.

Relationship between olfactomedin 4 staining and clinicopathologic characteristics. The relationship of olfactomedin 4 staining with clinicopathologic characteristics was investigated (Table I). The level of olfactomedin 4 immunoreactivity was first evaluated in tumor cells. When $>10 \%$ of tumor cells were stained, the immunostaining was considered positive for olfactomedin 4. In total, 59 (34\%) of the $176 \mathrm{CRC}$ cases were positive for olfactomedin 4. Olfactomedin 4-positive CRC cases showed earlier $\mathrm{T}$ classification $(\mathrm{P}=0.0180)$, $\mathrm{N}$ classification $(\mathrm{P}=0.0149)$ and stage $(\mathrm{P}=0.0144$, all by the Chi-square test) than olfactomedin 4-negative CRC cases (Table I). Olfactomedin 4 staining was not correlated with age, gender, tumor location, $\mathrm{M}$ classification, or histologic classification. We also examined the relation between survival and olfactomedin 4 staining in CRC. In the 176 
Table I. Correlation of olfactomedin 4 expression with clinicopathologic characteristics of $176 \mathrm{CRC}$ cases.

\begin{tabular}{|c|c|c|c|}
\hline & \multicolumn{2}{|c|}{ Olfactomedin 4 expression } & \multirow[b]{2}{*}{ P-value ${ }^{a}$} \\
\hline & Positive & Negative & \\
\hline Age & & & 0.9551 \\
\hline$\leq 65$ & $31(33 \%)$ & 62 & \\
\hline$>65$ & $28(34 \%)$ & 55 & \\
\hline Gender & & & 0.7943 \\
\hline Male & $36(34 \%)$ & 69 & \\
\hline Female & $23(32 \%)$ & 48 & \\
\hline Tumor location & & & 0.8075 \\
\hline Right/transverse & $12(35 \%)$ & 22 & \\
\hline Left/sigmoid/rectum & $47(33 \%)$ & 95 & \\
\hline T classification & & & 0.0180 \\
\hline $\mathrm{T} 1$ & $14(47 \%)$ & 16 & \\
\hline $\mathrm{T} 2$ & $15(39 \%)$ & 23 & \\
\hline $\mathrm{T} 3$ & $24(31 \%)$ & 54 & \\
\hline $\mathrm{T} 4$ & $6(20 \%)$ & 24 & \\
\hline $\mathrm{N}$ classification & & & 0.0149 \\
\hline N0 & $43(41 \%)$ & 63 & \\
\hline N1 & $16(23 \%)$ & 54 & \\
\hline M classification & & & 0.6085 \\
\hline M0 & $53(34 \%)$ & 102 & \\
\hline M1 & $6(29 \%)$ & 15 & \\
\hline Stage & & & 0.0144 \\
\hline I & $26(46 \%)$ & 31 & \\
\hline II & $16(36 \%)$ & 29 & \\
\hline III & $11(21 \%)$ & 42 & \\
\hline IV & $6(29 \%)$ & 15 & \\
\hline Histological classification & & & 0.1973 \\
\hline Well/moderately & $58(35 \%)$ & 110 & \\
\hline Poorly/mucinous & $1(13 \%)$ & 7 & \\
\hline
\end{tabular}

${ }^{a}$ Chi-square test.
CRC patients, those with olfactomedin 4-positive CRC had a better survival rate than patients with olfactomedin 4-negative CRC ( $\mathrm{P}=0.0092$, log-rank test) (Fig. 2A). It is well known that patients with $\mathrm{CRC}$ at stage I have a favorable rate of survival, whereas patients with CRC at stage IV show a poor rate of survival. However, it is difficult to predict the survival of patients with stage II or stage III CRC. Therefore, we analyzed the prognostic value of olfactomedin 4 in patients with stage II and III CRC. In stage II and III CRC patients $(\mathrm{n}=98)$, those with olfactomedin 4-positive CRC had a better survival rate than patients with olfactomedin 4-negative CRC ( $\mathrm{P}=0.0347$, log-rank test) (Fig. 2B). We then used Cox proportional hazards multivariate model to examine the association of clinicopathologic factors and expression of olfactomedin 4 with survival. Multivariate analysis indicated that $\mathrm{T}$ classification, $\mathrm{M}$ classification and olfactomedin 4 expression were independent predictors of survival in patients with CRC (Table II).

The level of olfactomedin 4 immunoreactivity was also evaluated in the tumor-associated stroma. Since extracellular staining of olfactomedin 4 at the invasive front was frequently observed, stromal olfactomedin 4 staining was considered positive when extracellular staining of olfactomedin 4 was stained at the invasive front. In total, 29 (16\%) of the 176 CRC cases were positive for stromal olfactomedin 4. Stromal olfactomedin 4 staining was not correlated with age, gender, tumor location, $\mathrm{T}$ classification, $\mathrm{N}$ classification, $\mathrm{M}$ classification, stage, or histologic classification (data not shown). In the 176 CRC patients, survival rate was not statistically different between patients with stromal olfactomedin 4-positive CRC and those with stromal olfactomedin 4-negative CRC (data not shown).

\section{Discussion}

Previously, we performed SAGE on four primary gastric cancers (4) and identified several gastric cancer-specific genes (5). Of these genes, olfactomedin 4 is a candidate gene for cancer-specific expression. In the present study, we examined the expression and distribution of olfactomedin 4 in CRC by immunohistochemistry and the relationship between
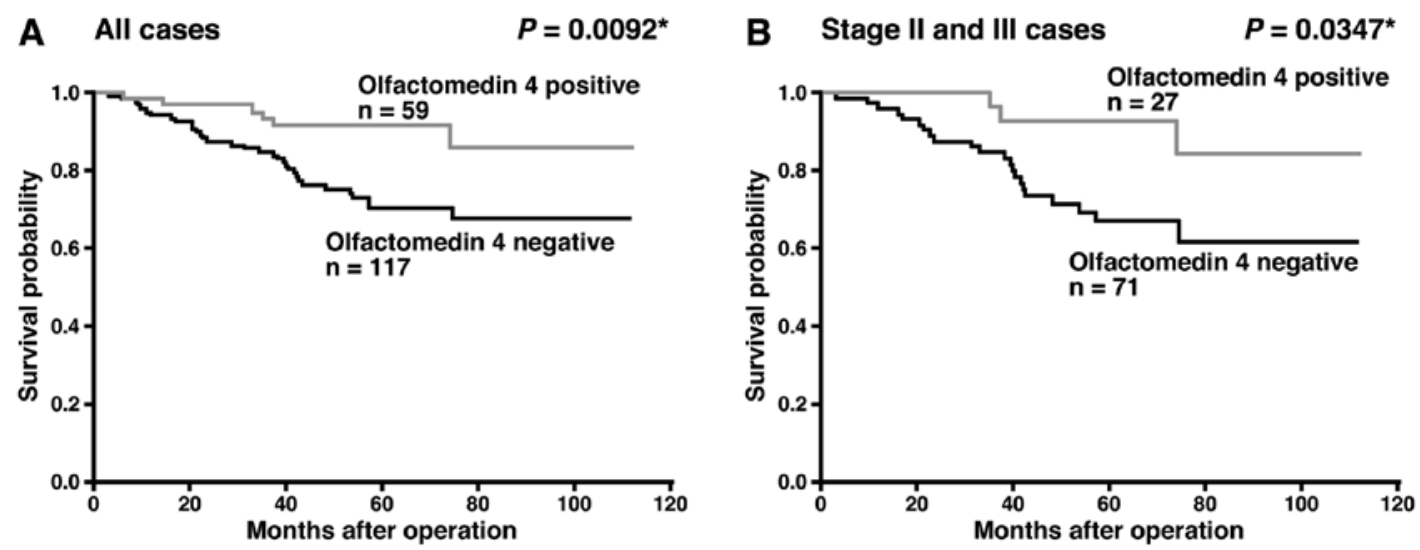

Figure 2. Survival of patients with CRC. (A) Kaplan-Meier curves of the CRC patients (all stages) with olfactomedin 4-negative or olfactomedin 4-positive tumors. (B) Kaplan-Meier curves of stage II and stage III CRC patients with olfactomedin 4-negative and olfactomedin 4-positive tumors. *Log-rank test. 
Table II. Multivariate analysis of factors that influence survival of patients with CRC.

\begin{tabular}{|c|c|c|c|c|}
\hline Factor & Hazard ratio & $(95 \% \mathrm{CI})$ & Chi-square test & $\mathrm{P}$-value \\
\hline Age & & & 1.586 & 0.2078 \\
\hline$\leq 65$ & 1 & (Reference) & & \\
\hline$>65$ & 1.511 & $(0.795-2.871)$ & & \\
\hline Gender & & & 0.708 & 0.4001 \\
\hline Male & 1 & (Reference) & & \\
\hline Female & 0.748 & $(0.381-1.470)$ & & \\
\hline Tumor location & & & 0.368 & 0.5441 \\
\hline Right/transverse & 1 & (Reference) & & \\
\hline Left/sigmoid/rectum & 0.745 & $(0.287-1.931)$ & & \\
\hline T classification & & & 5.995 & 0.0143 \\
\hline $\mathrm{T} 1 / 2$ & 1 & (Reference) & & \\
\hline $\mathrm{T} 3 / 4$ & 4.803 & $(1.367-16.869)$ & & \\
\hline $\mathrm{N}$ classification & & & 3.488 & 0.0618 \\
\hline No & 1 & (Reference) & & \\
\hline N1 & 7.327 & $(0.906-59.248)$ & & \\
\hline M classification & & & 11.161 & 0.0008 \\
\hline M0 & 1 & (Reference) & & \\
\hline M1 & 3.631 & $(1.704-7.737)$ & & \\
\hline Stage & & & 1.032 & 0.3097 \\
\hline $\mathrm{I} / \mathrm{II}$ & 1 & (Reference) & & \\
\hline III/IV & 3.254 & $(0.334-31.713)$ & & \\
\hline Histologic classification & & & 0.002 & 0.9632 \\
\hline Well/moderately differentiated & 1 & (Reference) & & \\
\hline Poorly differentiated /mucinous & 0.936 & $(0.194-4.773)$ & & \\
\hline Olfactomedin 4 expression & & & 4.486 & 0.0342 \\
\hline Positive & 1 & (Reference) & & \\
\hline Negative & 2.725 & $(1.078-6.890)$ & & \\
\hline
\end{tabular}

CI, Confidence interval. ${ }^{a}$ Cox proportional hazards model.

olfactomedin 4 staining and clinicopathologic characteristics. Although few epithelial cells in colonic mucosa distant from the CRC tissue showed olfactomedin 4 staining, strong and extensive olfactomedin 4 staining was found in $34 \%$ cases of $\mathrm{CRC}$, and olfactomedin 4-positive CRC cases showed earlier $\mathrm{T}$ classification, $\mathrm{N}$ classification and stage than olfactomedin 4-negative CRC cases. These results are consistent with results reported previously that olfactomedin 4 expression is up-regulated in early stage CRC and downregulated in advanced stage CRC (13). In our previous study in gastric cancer, olfactomedin 4-positive cases were found frequently in early stage cases (9). Taken together, expression of olfactomedin 4 is an early event, and loss/reduction of olfactomedin 4 expression is a late event in gastrointestinal malignancies.

It is generally accepted that apoptosis suppresses oncogenic transformation. The ability of tumor cell populations to expand in number is determined, not only by the rate of cell proliferation, but also by the rate of cell attrition. Apoptosis represents a major source of this attrition (16). Thus, resistance to apoptosis is a hallmark of most and perhaps all types of cancer. It has been reported that olfactomedin 4 interacts with GRIM-19 to attenuate retinoic acid and interferon $\beta$-mediated cellular apoptosis, and transient expression of olfactomedin 4 promoted tumor growth in C57/BL/6 mice (7). Therefore, expression of olfactomedin 4 may contribute to carcinogenesis by resistance to apoptosis at least in early stage CRC. In contrast, forced expression of olfactomedin 4 in an HT-29 cell line decreased cell adhesion and migration (13). Therefore, it is possible that loss/reduction of olfactomedin 4 expression induces tumor cell invasion in late stage CRC cases.

In the present study, univariate and multivariate analyses revealed that negative expression of olfactomedin 4 is a prognostic indicator. Furthermore, negative expression of olfactomedin 4 correlated with a short survival rate in stage II and III CRC cases. Patients diagnosed with stage II or III $\mathrm{CRC}$ have variable prognoses, and they are the group that would benefit most from discovery of a prognostic factor that can identify individuals for whom adjuvant treatment would be most advantageous. To clarify whether olfactomedin 4 immunostaining is useful for identification of patients most likely to benefit from adjuvant treatment, association between 
olfactomedin 4 staining and response to adjuvant therapies should be investigated.

In addition to cytoplasmic olfactomedin 4 staining, extracellular staining was also observed. Extracellular staining of olfactomedin 4 at the invasive front was frequently observed. Observation of the invasive front is important in the analysis of tumor cells, since it reflects the invasive potential of tumor cells. It has been reported that the expression of matrilysin in the invasive front is a promising biomarker predicting nodal metastasis of CRC (17). Overexpression of heparanase at the invasive front has been reported in gastric cancer and high expression of heparanase was a strong predictor of poor survival (18). These results indicate that the proteolytic degradation of the extracellular matrix by these molecules is one of the most important mechanisms in tumor progression, and the proteolytic degradation occurs at the invasive front. Although there was no correlation between stromal expression of olfactomedin 4 and clinicopathologic characteristics, stromal expression of olfactomedin 4 at the invasive front may partly contribute to the malignant behavior of CRC, such as local invasiveness.

Notably, extensive olfactomedin 4 staining was observed in the peritumoral mucosa of CRC, and olfactomedin 4 staining decreased gradually, moving away from the tumor tissue. It is well known that the peritumoral mucosa of CRC is often hyperplastic, and various growth factors, such as transforming growth factor- $\alpha$ and basic fibroblast growth factor, are increased in the peritumoral mucosa (19). Since expression of olfactomedin 4 in the crypt epithelium of inflamed colonic mucosa has been reported (20), olfactomedin 4 expression may be induced by growth factors and may function as an antiapoptotic factor in the peritumoral mucosa of CRC.

In summary, we showed that olfactomedin 4 is a valuable marker for long survival in patients with CRC. However, the significance of extracellular staining of olfactomedin 4 at the invasive front and extensive olfactomedin 4 staining in the peritumoral mucosa of CRC remains unclear. Since olfactomedin 4 is a secreted protein, identification of a cell surface receptor for olfactomedin 4 will further improve our understanding of the basic biology of olfactomedin 4 .

\section{Acknowledgements}

We thank Mr Shinichi Norimura for the excellent technical assistance and advice. We thank the Analysis Center of Life Science, Hiroshima University for the use of their facilities. This work was supported, in part, by Grants-in-Aid for Cancer Research from the Ministry of Education, Culture, Science, Sports and Technology of Japan, in part, by a Grant-in-Aid for the Third Comprehensive 10-Year Strategy for Cancer Control and for Cancer Research from the Ministry of Health, Labour and Welfare of Japan and in part by a grant (07-23911) from the Princess Takamatsu Cancer Research Fund.

\section{References}

1. Popat S, Hubner $\mathrm{R}$ and Houlston RS: Systematic review of microsatellite instability and colorectal cancer prognosis. J Clin Oncol 23: 609-618, 2005.

2. Oue N, Kuniyasu H, Noguchi T, et al: Serum concentration of Reg IV in patients with colorectal cancer: overexpression and high serum levels of Reg IV are associated with liver metastasis. Oncology 72: 371-380, 2007.

3. Kobayashi T, Hino S, Oue N, Asahara T, Zollo M, Yasui W and Kikuchi A: Glycogen synthase kinase 3 and h-prune regulate cell migration by modulating focal adhesions. Mol Cell Biol 26: 898-911, 2006.

4. Oue N, Hamai Y, Mitani Y, et al: Gene expression profile of gastric carcinoma: identification of genes and tags potentially involved in invasion, metastasis and carcinogenesis by serial analysis of gene expression. Cancer Res 64: 2397-2405, 2004.

5. Aung PP, Oue N, Mitani Y, et al: Systematic search for gastric cancer-specific genes based on SAGE data: melanoma inhibitory activity and matrix metalloproteinase-10 are novel prognostic factors in patients with gastric cancer. Oncogene 25: 2546-2557, 2006.

6. Zhang J, Liu WL, Tang DC, et al: Identification and characterization of a novel member of olfactomedin-related protein family, hGC-1, expressed during myeloid lineage development. Gene 283: 83-93, 2002.

7. Zhang X, Huang Q, Yang Z, Li Y and Li CY: GW112, a novel antiapoptotic protein that promotes tumor growth. Cancer Res 64: 2474-2481, 2004.

8. Liu W, Zhu J, Cao L and Rodgers GP: Expression of hGC-1 is correlated with differentiation of gastric carcinoma. Histopathology 51: 157-165, 2007.

9. Oue N, Sentani K, Noguchi T, et al: Serum olfactomedin 4 (GW112, hGC-1) in combination with Reg IV is a highly sensitive biomarker for gastric cancer patients. Int J Cancer 125 : 2383-2392, 2009.

10. Koshida S, Kobayashi D, Moriai R, Tsuji N and Watanabe N: Specific overexpression of OLFM4 (GW112/HGC-1) mRNA in colon, breast and lung cancer tissues detected using quantitative analysis. Cancer Sci 98: 315-320, 2007.

11. Conrotto P, Roesli C, Rybak J, et al: Identification of new accessible tumor antigens in human colon cancer by ex vivo protein biotinylation and comparative mass spectrometry analysis. Int $\mathrm{J}$ Cancer 123: 2856-2864, 2008.

12. Van der Flier LG, Haegebarth A, Stange DE, van de Wetering M and Clevers H: OLFM4 is a robust marker for stem cells in human intestine and marks a subset of colorectal cancer cells. Gastroenterology 137: 15-17, 2009.

13. Liu W, Liu Y, Zhu J, Wright E, Ding I and Rodgers GP: Reduced hGC-1 protein expression is associated with malignant progression of colon carcinoma. Clin Cancer Res 14: 1041-1049, 2008.

14. Sobin LH and Wittekind $\mathrm{CH}$ (eds): TNM Classification of Malignant Tumors. 6th edition. Wiley-Liss, New York, pp65-68, 2002.

15. Mantel N: Evaluation of survival data and two new rank order statistics arising in its consideration. Cancer Chemother Rep 50: 163-170, 1966.

16. Hanahan D and Weinberg RA: The hallmarks of cancer. Cell 100: 57-70, 2000.

17. Kurokawa S, Arimura Y, Yamamoto H, et al: Tumour matrilysin expression predicts metastatic potential of stage I (pT1) colon and rectal cancers. Gut 54: 1751-1758, 2005.

18. Takaoka M, Naomoto Y, Ohkawa T, et al: Heparanase expression correlates with invasion and poor prognosis in gastric cancers. Lab Invest 83: 613-622, 2003.

19. Kuniyasu H, Yasui W, Shinohara H, et al: Induction of angiogenesis by hyperplastic colonic mucosa adjacent to colon cancer. Am J Pathol 157: 1523-1535, 2000.

20. Shinozaki S, Nakamura T, Iimura M, et al: Upregulation of Reg 1 alpha and GW112 in the epithelium of inflamed colonic mucosa. Gut 48: 623-629, 2001. 